\title{
The United Kingdom's global health funding cuts will exacerbate inequities
}

To the Editor - We write to express our dismay over the United Kingdom government's recent decision to cut overseas development assistance and its funding of health and development research in low- and middle-income countries (LMICs) ${ }^{1,2}$.

As academics working in the field of global health, we and our colleagues in Africa, Latin America and Asia are facing the abrupt termination and/or disruption of existing research projects. Coming as it does in the midst of the COVID-19 global health crisis, such an extreme reduction in financial support will not only harm research partnerships and scientific endeavours that have taken years to establish, but will also damage the United Kingdom's global health reputation.

The scale of cuts being proposed is such that universities in the United Kingdom will have to renege on agreements and contracts in a way that will be distasteful and unethical. Trust and equitable partnerships are the cornerstone of successful international research, and the decision to withdraw funding from existing projects will cause irreparable damage.

Of particular concern is the effect that this sudden reduction in funding will have on capacity building, which was a key goal of the Global Challenges Research Fund (GCRF) ${ }^{3}$. Many LMICs, with which the United Kingdom has strong historical and cultural ties, have fragile research communities that have been slowly and carefully growing with the help of the GCRF. This is now under threat.

Although we understand that the United Kingdom government faces financial challenges caused by the pandemic, this sudden withdrawal of funds to support health improvement in LMICs will exacerbate inequalities and jeopardize LMIC research ecosystems at a time when solutions to global challenges have never been more needed.

\author{
Victoria Bird, Feng J. He, Paul Heritage, \\ Paul Kelly $\mathbb{} \mathbb{D}^{凶}$, Graham MacGregor, \\ Adrian Martineau, David McCoy, \\ Doreen Montag, Andrew J. Prendergast, \\ Stefan Priebe, Giuliano Russo and \\ Francois van Loggerenberg (D) \\ Queen Mary University of London, London, UK. \\ $\bigotimes_{e-m a i l: m . p . k e l l y @ q m u l . a c . u k}$
}

Published online: 12 April 2021

https://doi.org/10.1038/s41564-021-00905-Z

References

1. Smith, C. UKRI Official Development Assistance letter 11 March 2021. UK Research and Innovation https://go.nature. com/3udBcY0 (2021).

2. Thornton, J. Researchers slam UK cuts to global health research. Lancet 397, 1168-1170 (2021).

3. Global Challenges Research Fund. UK Research and Innovation https://go.nature.com/3cKxpeV (2021).

Author contributions

A.J.P. wrote the first draft of the Correspondence, which was edited by P.K., D.M.C. and all other authors.

Competing interests

$\square \quad$ The authors declare no competing interests. 